\title{
The role of adiponectin in renal physiology and development of albuminuria
}

\author{
Georgios A Christou and Dimitrios N Kiortsis \\ Laboratory of Physiology, Medical School, University of Ioannina, 45110 loannina, Greece
}

Correspondence should be addressed to D N Kiortsis

Email

dkiorts@cc.uoi.gr

\begin{abstract}
Adiponectin is secreted by the adipose tissue and is downregulated in states of obesity and insulin resistance. There is a growing body of evidence indicating that adiponectin has renoprotective effects and protects against the development of albuminuria in rodent experiments. Adiponectin crossing the glomerular filtration barrier possibly inhibits inflammation, fibrosis and oxidative stress in kidneys through activation of AMP-activated protein kinase. Moreover, microalbuminuria is a well established early sign of progressive cardiovascular and renal disease, even in subjects with preserved glomerular filtration rate. Studies investigating the relationship between serum adiponectin levels and urinary albumin excretion rate (UAE) have yielded conflicting data and the mechanisms underlying the interplay between adiponectin and albuminuria remain to be elucidated. This article constitutes a critical review attempting to clarify any remaining confusion about this matter. Furthermore, this article examines the clinical significance of adiponectin-albuminuria interplay, suggesting that adiponectin is possibly involved in the development of albuminuria that is associated with obesity, diabetes and cardiovascular disease and may mediate, at least in part, the actions of medical treatments that influence UAE, such as angiotensin-converting-enzyme inhibitors, angiotensin II receptor blockers, thiazolidinediones, fenofibrate and diet. Further studies to investigate more thoroughly the renoprotective role of adiponectin in the human setting should be carefully planned, focusing on causality and the possible influence of adiponectin on the development of albuminuria in specific clinical settings.
\end{abstract}

Journal of Endocrinology (2014) 221, R49-R61

\section{Introduction}

Adiponectin is an adipokine secreted by the adipocytes and downregulated in obesity (Hotta et al. 2000). Low levels of circulating adiponectin appear to contribute to the pathophysiology of insulin resistance, type 2 diabetes and cardiovascular disease (CVD) in obese or overweight patients (Hotta et al. 2000). Adiponectin is currently considered to be eliminated through renal biodegradation/ excretion, but this mechanism has been proposed mostly on the basis of indirect data (von Eynatten et al. 2009). Adiponectin has been reported to exert beneficial renal effects, protecting against the development of albuminuria in rodent experiments (Ohashi et al. 2007, Sharma et al. 2008, Nakamaki et al. 2011). Adiponectin has been shown to have anti-inflammatory, antifibrotic and antioxidant effects on kidneys through AMP-activated protein kinase (AMPK) activation. The influence of 
adiponectin on the development of albuminuria appears to have high clinical significance, because microalbuminuria constitutes an early sign of progressive cardiovascular and renal diseases in subjects with and without diabetes (de Jong \& Curhan 2006). At the time that microalbuminuria develops the glomerular filtration rate (GFR) is typically normal or only modestly elevated. In addition, the development of albuminuria can be associated with minimal structural glomerular changes but without any significant change in renal haemodynamics.

Several studies have been performed to investigate the relationship between serum adiponectin levels and urinary albumin excretion rate (UAE), but the results appear to be conflicting. It is well-known that albuminuria is associated with diabetes and obesity and that increasing albuminuria constitutes an adverse cardiovascular risk factor in these states (Gerstein et al. 2000, Kiortsis \& Christou 2012). Taking into account that adiponectin has been found to play an important role in the pathophysiology of diabetes, obesity and CVD, the elucidation of the relationship between the patterns of circulating adiponectin and UAE and of the pathophysiological interplay between adiponectin and albuminuria would help to clarify the exact role of adiponectin in the development of albuminuria that is associated with diabetes, obesity and CVD. Moreover, adiponectin is found to be involved in the pathways of medical treatments that influence UAE, such as angiotensin converting-enzyme inhibitors (ACE-I), angiotensin II receptor blockers (ARBs), thiazolidinediones, fenofibrate and diet. In this context, this article presents a critical review of the relevant studies in order to evaluate the possible role of adiponectin in renal physiology and development of albuminuria in the clinical setting. A literature search based on PubMed listings up to 3 December 2013 using adiponectin as the search term identified 11827 articles, the abstracts of which were read. Only the papers reporting on the role of adiponectin in renal physiology and development of albuminuria were considered eligible and were read in full for inclusion in the review.

\section{Plasma adiponectin levels}

Plasma adiponectin levels are usually measured with ELISA kits. In healthy human individuals, plasma adiponectin levels increase with increasing age and an elevation of $\sim 1 \mathrm{mg} / \mathrm{l}$ of circulating adiponectin has been reported for every 10 years of age (Obata et al. 2013). Moreover, plasma adiponectin levels are higher in women than in men (Obata et al. 2013). Taking into account the wide range of variability of circulating adiponectin in healthy humans, strict cutoffs cannot be defined. However, a crude estimation of plasma adiponectin levels in non-elderly healthy individuals is between 4 and $10 \mathrm{mg} / \mathrm{l}$ for males and between 6 and $15 \mathrm{mg} / \mathrm{l}$ for females (Isobe et al. 2005, Obata et al. 2013).

\section{Renal elimination of adiponectin}

Adiponectin is cleared rapidly from the circulation primarily from the liver, involving at least in part the biliary route, and secondarily from the kidneys (Tacke et al. 2005, Halberg et al. 2009). Adiponectin monomers $(28 \mathrm{kDa})$ and dimers have a molecular weight small enough to cross the normal functioning glomerular filtration barrier and thereby adiponectin can be traced in the urine of healthy subjects (Shimotomai et al. 2005). Adiponectin is normally present on the endothelium and in the smooth muscle cells of intrarenal arteries/arterioles and on the endothelium of glomerular and peritubular capillaries, while a weaker signal for adiponectin can be found in proximal and distal tubular epithelial cells (Rovin et al. 2005, von Eynatten et al. 2009, Perri et al. 2013). Minimal proteolytic cleavage of glomerular endotheliumbound adiponectin is believed to cause degradation of high-order complexes of adiponectin and subsequent lowgrade excretion of adiponectin monomers and dimers in the urine of healthy subjects (von Eynatten et al. 2009). Proximal tubular cells can produce and secrete adiponectin, which may contribute to some extent to the total amount of urinary adiponectin (Perri et al. 2013). Notably, although urinary adiponectin of normal humans has been reported to be below the limit of detection with ELISA kits, low-intensity signals of urinary adiponectin have been detected with western blotting (Rovin et al. 2005, von Eynatten et al. 2009). Although monomers and dimers are the only isoforms of adiponectin detected in urine of healthy subjects, in patients with albuminuria trimers of adiponectin (i.e. low-molecular-weight adiponectin) are also excreted to a considerable extent (Koshimura et al. 2004, Rovin et al. 2005, von Eynatten et al. 2009). Therefore, in patients with albuminuria monomers, dimers and trimers of adiponectin appear to constitute the dominant isoforms of urinary adiponectin. Highmolecular-weight (HMW) adiponectin has been reported to be minimally detected in urine of patients with proteinuria, possibly due to the leakage of HMW adiponectin through a dysfunctional glomerular filtration barrier (Shen et al. 2008). This currently proposed route of renal biodegradation/excretion of adiponectin is mostly

Published by Bioscientifica Ltd 
derived on the basis of indirect experimental data. Further studies are needed to investigate the exact pathways of renal elimination of adiponectin.

\section{The role of adiponectin in renal physiology}

It has been found that adiponectin receptor ADIPOR1 and catalytic AMPK subunits are expressed by the four cell types constituting the glomerulus, namely endothelial cells, podocytes, mesangial cells and Bowman's capsule epithelial cells, as well as by proximal tubular cells (Cammisotto \& Bendayan 2008, Shen et al. 2008, Perri et al. 2013). However, the adiponectin receptor ADIPOR2 has been reported not to be expressed to a substantial extent by glomerular and proximal tubular cells (Cammisotto \& Bendayan 2008, Sharma et al. 2008, Perri et al. 2013). It is well known that ADIPOR1 has a high affinity for the globular form of adiponectin (i.e. a fragment containing the globular domain of adiponectin) and can activate AMPK (Cammisotto \& Bendayan 2008). Adiponectin crossing the glomerular filtration barrier possibly acts on glomerular cells causing an ADIPOR1mediated activation of AMPK. AMPK is a heterotrimeric protein consisting of a combination of $\alpha_{1 / 2}, \beta_{1 / 2}$, and $\gamma_{1 / 2 / 3}$ subunits with the $\alpha_{1}$ rather than $\alpha_{2}$ subunit expressed in the kidney (Lee et al. 2007). The enzymatic activity of AMPK is dependent on phosphorylation of Thr172 of the $\alpha$-subunit. The AMPK pathway appears to be necessary for the maintenance of normal renal physiology (Lee et al. 2007). The AMPK can activate ATP-generating processes, such as fatty acid oxidation, while shutting down energyconsuming pathways. Moreover, AMPK normally inhibits protein synthesis and its suppression appears to mediate high-glucose-induced protein synthesis. AMPK is also a potent oxidative stress inhibitor, controlling the generation of reactive oxygen species (ROS; Ouedraogo et al. 2006). These data indicate that adiponectin may control anabolic processes (such as cell division) and oxidative stress in glomerulus through AMPK-activated pathways and thus could contribute to the maintenance of normal renal function. The following experimental data further support this statement.

Sharma et al. (2008) demonstrated that adiponectin knockout $\left(\mathrm{Ad}^{-/-}\right)$mice exhibited increased albuminuria compared with WT mice, while adiponectin administration normalised albuminuria in $\mathrm{Ad}^{-/-}$mice. Podocyte foot processes were segmentally fused in the $\mathrm{Ad}^{-/-}$ glomeruli, while adiponectin administration restored podocyte foot processes in $\mathrm{Ad}^{-1-}$ mice. Importantly, albumin permeability across a differentiated podocyte cell monolayer was reduced by the addition of adiponectin in vitro. These data imply that the protective role of adiponectin against the development of albuminuria occurs, at least in part, through a direct action of adiponectin on podocytes independently of any systemic effects of adiponectin. This regulation may involve adiponectin-induced activation of AMPK pathway in podocytes, because 5-aminoimidazole-4-carboxamide$1-\beta$-D-ribonucleoside (AICAR), which is a specific activator of AMPK, was found to reduce permeability of podocytes to albumin, while a specific inhibitor of AMPK, adenine 9- $\beta$-D-arabinofuranoside (ARA), increased permeability to albumin. This mechanism is further supported by the evidence that AICAR administration restored albuminuria in $\mathrm{Ad}^{-1-}$ mice. Furthermore, the beneficial effects of adiponectin-induced activation of AMPK on podocyte function may involve the promotion of normal localisation of zona occludens-1 (ZO1) along the plasma membrane of podocytes. ZO1 is associated with tight junction adherence and normal function of the slit diaphragm. Indeed, Sharma et al. (2008) showed that ARA induced a redistribution of $\mathrm{ZO} 1$, causing a translocation of ZO1 away from the plasma membrane to the cytosol, whereas both adiponectin and AICAR restored the linear membrane localisation of $\mathrm{ZO} 1$ in podocytes. In addition, increased levels of markers of renal oxidant stress were found in $\mathrm{Ad}^{-1-}$ mice. These changes were restored by adiponectin treatment. These findings indicate that the reduction of oxidant stress is possibly a pathway by which adiponectin and AMPK activation provide protection against albuminuria and increased podocyte permeability.

Rutkowski et al. (2013) investigated the renoprotective properties of adiponectin in podocyte apoptosis through targeted activation of caspase-8 (POD-ATTAC) mice generated by induction of caspase-8-mediated apoptosis in podocytes after injection of a construct-specific agent. POD-ATTAC mice exhibited kidney damage that recovered after the initial induction. $\mathrm{Ad}^{-1-}$ POD-ATTAC mice had more severe albuminuria and ablated podocytes failed to recover. Adiponectin-overexpressing POD-ATTAC mice maintained normal GFR, had reduced renal interstitial fibrosis and exhibited a greater recovery from podocyte injury and albuminuria. These findings imply that adiponectin aids in restoring podocytes and renal function but the initial protection of podocytes from apoptosis may be minimal.

Nakamaki et al. (2011) examined streptozotocininduced diabetic rats that received injections of an adenovirus to cause overexpression of adiponectin (Ad-Adipo) or control protein (Ad-lacZ). Ad-Adipo rats

Published by Bioscientifica Ltd. 
exhibited reduced proteinuria compared with Ad-lacZ rats. In addition, Ad-Adipo rats had decreased transforming growth factor $\beta 1$ (TGF- $\beta 1$ ) levels in the renal cortex. Taking into account the involvement of TGF- $\beta 1$ in the pathogenesis of diabetic nephropathy, contributing to cellular hypertrophy and increased synthesis of collagen, adiponectin may inhibit the adverse TGF- $\beta 1$-mediated changes in renal physiology, that result in development of proteinuria. Nakamaki et al. (2011) also demonstrated that Ad-Adipo rats had increased levels of nephrin-mRNA in renal cortex. Nephrin is a protein found in podocytes and is crucial for the proper function of the glomerular filtration barrier, whereas downregulation of nephrin contributes to the development of proteinuria in diabetic nephropathy. Thus, the upregulation of nephrin levels in renal cortex appears to constitute a pathway through which adiponectin can reduce albuminuria, at least in diabetic nephropathy. Furthermore, this study showed that Ad-Adipo rats had decreased proinflammatory endothelin-1, plasminogen activator inhibitor-1 and inducible nitric oxide synthase (iNOS) mRNA levels and increased vasodilatory endothelial nitric oxide synthase (eNOS) mRNA levels in renal cortex. Therefore, adiponectin appears to reduce endothelial dysfunction in diabetic nephropathy.

Ohashi et al. (2007) reported that adiponectin can attenuate the adverse effects of partial nephrectomy on renal structure and function, eventually reducing the albuminuria associated with this procedure. Partial nephrectomy results in hypertrophy of the remaining nephrons, podocyte injury, glomerular fibrosis and development of proteinuria through adaptive glomerular hyperfiltration, oxidative stress and inflammation. In the relevant study, subtotal nephrectomy was performed in $\mathrm{Ad}^{-/-}$mice and WT mice. $\mathrm{Ad}^{-/-}$mice exhibited more severe glomerular hypertrophy, glomerular infiltration of macrophages, glomerular and tubulointerstitial fibrosis and decreased nephrin-mRNA levels in the remnant kidney, and increased albuminuria compared with WT mice. Exogenous adiponectin replenishment of $\mathrm{Ad}^{-1-}$ mice resulted in reduction in albuminuria and reversal of the adverse changes in renal structure of $\mathrm{Ad}^{-/-}$mice mentioned earlier. Therefore, adiponectin appears to attenuate albuminuria through activation of antiinflammatory, antifibrotic and antioxidative pathways. Moreover, in this study immunohistochemical analysis revealed abundant immunostaining for adiponectin in the glomeruli and interstitium of the remnant kidney of WT mice after subtotal nephrectomy, but limited glomerular and tubulointerstitial immunostaining for adiponectin in nonoperated WT mice. This finding indicates that adiponectin possibly accumulates in the glomeruli and interstitium of the remnant kidney to counteract this renal injury, in response to an as yet unknown signal from the injured kidney. Further studies are needed to elucidate this issue.

In addition, adiponectin was reported to attenuate the adverse renal effects of angiotensin II (Fang et al. 2013). Specifically, adiponectin was found to reduce angiotensin II-induced oxidative stress (NADPH oxidase activation), inflammation (NF-kB activity), and fibrosis (fibronectin expression) in human renal tubular cells. Adiponectin was shown to inhibit angiotensin II-induced activation of NADPH oxidase via the ADIPOR1-mediated activation of both AMPK and cAMP-Epac pathways.

The above-mentioned studies demonstrating the beneficial effects of adiponectin regarding the reduction in albuminuria were carried out exclusively in rodent experimental settings. The relevance to the human situation of studies in rodents should be interpreted with caution. Thus, further studies are needed to demonstrate the protective role of adiponectin against albuminuria in human experimental settings. The above-mentioned studies should be considered separately from studies investigating the role of adiponectin in the setting of acute renal injury. The latter group of studies includes two trials investigating whether adiponectin can modulate the adverse effects of renal ischemia-reperfusion injury in mice (Cheng et al. 2012, Jin et al. 2013). These studies yielded conflicting results with regard to the acute responses of various parameters of renal structure and function resulting from acute renal injury. Acute responses of this type are minimally predictable, depend to a great extent on the time interval between the initial injury and the studied response and are influenced by postoperative stress. Thus the assessment of the possible impact of adiponectin on these responses may lead to false conclusions regarding the role of adiponectin in renal physiology.

The concept of the protective role of adiponectin against the development of albuminuria is reinforced by data from a genetic study performed by Kacso et al. (2012a). The presence of T allele at 276GT polymorphism of adiponectin gene is associated with increased circulating adiponectin (Qi et al. 2005). Kacso et al. (2012a) reported that in subjects with type 2 diabetes mellitus and albuminuria, the GG genotype at 276GT was more frequent and the prevalence of the $\mathrm{T}$ allele $(\mathrm{GT}+\mathrm{TT}$ genotypes) was lower compared with patients with type 2 diabetes mellitus and normoalbuminuria. Therefore, adiponectin may protect against the development of

Published by Bioscientifica Ltd 
albuminuria in subjects with genetically determined increased levels of serum adiponectin. It is well known that the protective roles of adiponectin and AMPK on endothelial cells involve neutralisation of ROS, reduction in adhesion molecules synthesis and suppression of cell proliferation (Rakatzi et al. 2004, Davis et al. 2006, Ouedraogo et al. 2006). Importantly, high levels of ROS, increased expression of adhesion molecules and hypercellularity constitute markers of glomerulonephropathies. These data imply that adiponectin crossing the glomerular filtration barrier may control oxidative stress and cell division in glomerulus through ADIPOR1-mediated activation of AMPK (Fig. 1). This regulation could explain at least in part the adiponectin-induced reduction in albuminuria through the beneficial effects of adiponectin on glomerular structure and function.

\section{Urinary adiponectin excretion rate in patients with albuminuria}

In steady-state subjects, in whom plasma adiponectin levels do not change over time, urinary adiponectin excretion rate (as assessed by the ratio urinary adiponectin:urinary creatinine) equates with adiponectin production rate in adipose tissue - hepatic adiponectin excretion rate. The urinary adiponectin excretion rate has been reported to be positively correlated with UAE and negatively with GFR (Koshimura et al. 2004, Fujita et al. 2006, Jorsal et al. 2013). The positive relationship between urinary adiponectin excretion rate and UAE has been shown to strengthen with increasing UAE; this association has been found to exist mainly in patients with macroalbuminuria (UAE $>300 \mathrm{mg} / 24 \mathrm{~h}$ ) or microalbuminuria (UAE between 30 and $300 \mathrm{mg} / 24 \mathrm{~h}$ ) and less consistently in subjects with normoalbuminuria (Koshimura et al. 2004, Fujita et al. 2006, Jorsal et al. 2013). Thus, in patients with increased UAE or decreased GFR, the adiponectin production rate - hepatic adiponectin excretion rate appears to be increased. This elevation could be attributed to an increased rate of adiponectin production, because adiponectin expression and adiponectin protein content in visceral as well as subcutaneous adipose tissue were found to be increased in patients with end-stage renal disease (Martinez Cantarin et al. 2013). Studies investigating the possible modulation of hepatic adiponectin excretion rate in patients with renal disease are lacking, and thus further studies investigating this issue are needed. Taking into account the renoprotective properties of adiponectin mentioned earlier, a plausible explanation for the upregulation of adipose tissue adiponectin production in patients with albuminuria or decreased GFR is that adiponectin production in adipose tissue and

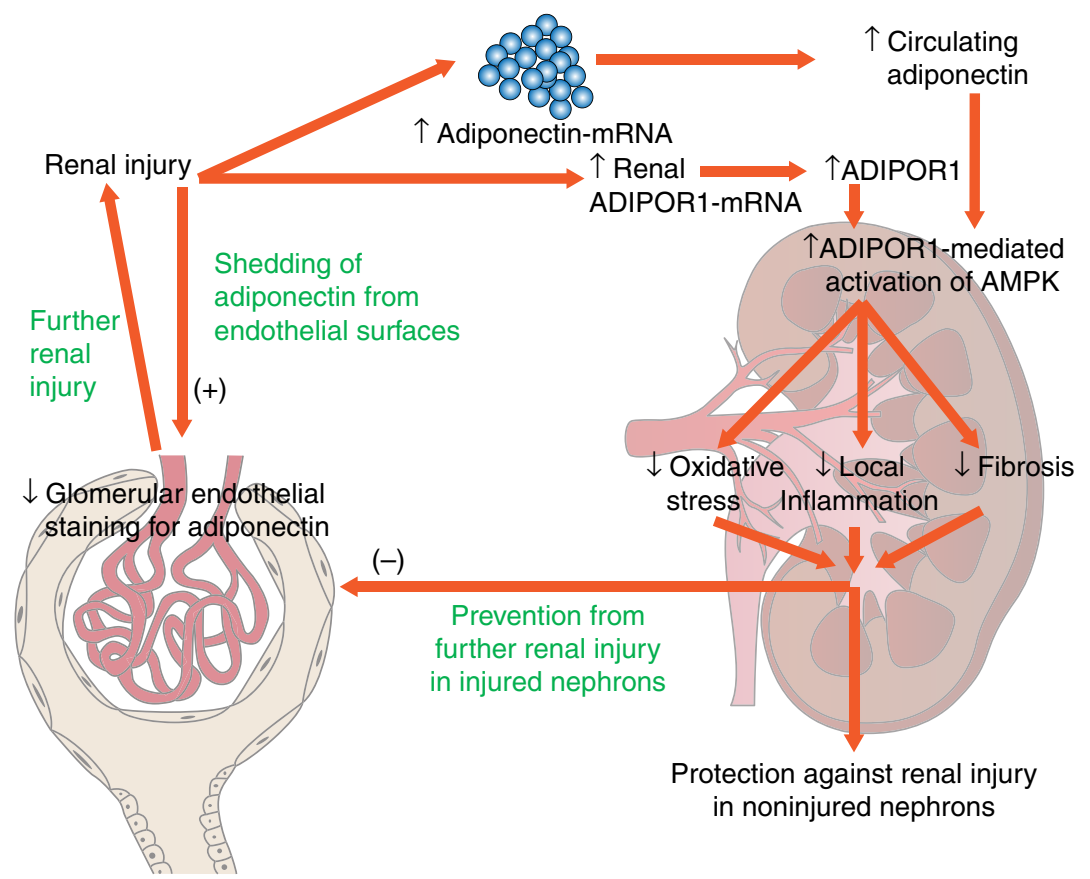

\section{Figure 1}

The proposed role of adiponectin in renal pathophysiology and the interplay between adiponectin and renal injury. AMPK, AMP-activated protein kinase; ADIPOR1, adiponectin receptor 1. A full colour version of this figure is available at http://dx.doi.org/10.1530/JOE-13-0578

http://joe.endocrinology-journals.org DOI: 10.1530/JOE-13-0578
(C) 2014 Society for Endocrinology Printed in Great Britain
Published by Bioscientifica Ltd 
secretion into the bloodstream is enhanced via an as yet unknown mechanism to mitigate renal injury. Consistently, Ohashi et al. (2007) found that adiponectin accumulated in the glomeruli and interstitium of the remnant kidney of WT mice to counteract for the subtotal nephrectomy-induced renal injury. In addition, serum adiponectin levels have been found to be positively correlated with urinary levels of markers of renal tubulointerstitial injury in diabetics, such as $\mathrm{N}$-acetylglucosaminidase and MCP1 (Fujita et al. 2006). Moreover, exposure of proximal tubular cells to recombinant adiponectin has been reported to result in decreased MCP1 secretion (Shen et al. 2008).

\section{Serum adiponectin levels in patients with albuminuria}

Serum adiponectin levels can be found by using the following equation

$$
\begin{aligned}
\text { Serum adiponectin }= & \text { urinary adiponectin excretion } \\
& \text { rate/renal adiponectin clearance } .
\end{aligned}
$$

Furthermore, renal clearance of adiponectin is expected to increase with both increasing GFR and increasing UAE (increased leakage of adiponectin through a dysfunctional glomerular-filtration barrier). Hence, in subjects with normoalbuminuria, taking into account the finding that urinary adiponectin excretion rate is mildly increased or even decreased as mentioned earlier, serum adiponectin levels are expected to decrease with increasing UAE (increasing renal adiponectin clearance). Consistently, serum adiponectin levels have been reported to be negatively correlated with UAE mainly in subjects with normoalbuminuria and less consistently in patients with low-grade microalbuminuria (Tsioufis et al. 2005, Sharma et al. 2008, Barlovic et al. 2009, 2010, Tamba et al. 2010). Importantly, this relationship was reported in populations with preserved GFR, without the concomitant upregulation of circulating adiponectin due to reduced GFR. This pattern of an inverse association between circulating adiponectin and UAE can be also attributed to the protective role of adiponectin against the development of albuminuria. In this aspect, the reported negative relationship between serum adiponectin levels and UAE in subjects with normoalbuminuria could be explained firstly by the increased renal clearance of adiponectin in subjects with relatively increased UAE (even though in the normal range) and secondly by the low likelihood of individuals with high serum adiponectin levels developing an elevation of UAE. On the other hand, serum adiponectin levels have been found to be increased in subjects with macroalbuminuria or less consistently in patients with microalbuminuria and to be positively correlated with UAE in these patients (Koshimura et al. 2004, Looker et al. 2004, Costacou et al. 2005, Frystyk et al. 2005, Fujita et al. 2006, Schalkwijk et al. 2006, Kacso et al. 2012b). These studies included mostly diabetics with reduced GFR. In patients with macroalbuminuria or microalbuminuria, the considerable upregulation of urinary adiponectin excretion rate, despite the increased UAE (and thus increased renal adiponectin clearance), results in increased serum adiponectin levels, as indicated by equation (1). In these patients, the dominant factor influencing serum adiponectin levels appears to be the elevated urinary adiponectin excretion rate (which is caused by increased adipose tissue adiponectin production), rather than UAE. The reported upregulation of circulating adiponectin in states of overt nephropathy may represent, at least in part, a physiological response to prevent further progression of renal injury. This mechanism possibly coexists with the upregulation of circulating adiponectin due to decreased renal clearance of adiponectin in subjects with advanced albuminuria and concomitant decreased GFR. In patients with albuminuria and decreased GFR, serum adiponectin levels are expected to be higher than those in patients with an equal grade of albuminuria and preserved GFR. Therefore, the circulating adiponectin-UAE relationship appears to change during the course of the development of albuminuria. First, high levels of serum adiponectin are associated with decreased $\mathrm{UAE}$, but this association is later reversed, probably at the level of microalbuminuria. Further prospective studies are needed to confirm this threshold point. The upregulation of the adiponectin production rate as a compensatory mechanism to mitigate further renal injury appears to be the main cause of increased serum adiponectin levels in patients with advanced albuminuria. As mentioned above, the elevation of adiponectin expression and production in the setting of human renal disease has been demonstrated only in patients with end-stage renal disease (Martinez Cantarin et al. 2013). Although, Komura et al. (2010) reported that adiponectin production in the adipose tissue of subtotal nephrectomised mice did not change at 1 month after operation, the time duration of 1 month may be too short to elicit a full-blown upregulation of adiponectin production. Moreover, Jian et al. (2013) reported a decrease in adiponectin expression in adipose tissue of subtotally nephrectomised rats, but subtotal nephrectomy was accompanied by changes in fat

Published by Bioscientifica Ltd 
mass and insulin sensitivity, that could interfere with the altered adiponectin expression in adipose tissue. Further studies are needed to confirm the elevation of adiponectin expression and production by human adipose tissue in response to various types of renal disease and to investigate the possible existence of signaling molecules that could transmit these changes.

With regard to the relationship between serum adiponectin and proteinuria, consistent results with serum adiponectin-albuminuria association have been reported. Macroalbuminuria practically equals proteinuria with urinary protein excretion (UPE) $>500 \mathrm{mg} / 24 \mathrm{~h}$ and characterises overt nephropathy. Therefore, patients with proteinuria are expected to have increased serum adiponectin levels compared with nonproteinuric individuals and a positive circulating adiponectin-UPE relationship has been consistently reported in patients with proteinuria (Zoccali et al. 2003, Menon et al. 2006, Takahashi et al. 2007, Hayakawa et al. 2009, Kamimura et al. 2012). However, in some studies including patients with type 2 diabetes, subjects with proteinuria had decreased circulating adiponectin compared with patients without proteinuria and a negative circulating adiponectin-UPE relationship was found (Yilmaz et al. 2004, Yenicesu et al. 2005). In these studies, the type 2 diabetics with proteinuria had increased insulin resistance compared with the diabetics without proteinuria and this may account for the reported downregulation of serum adiponectin levels in patients with type 2 diabetes and proteinuria.

Regarding the role of adiponectin subfractions in renal physiology, circulating HMW adiponectin appears to be elevated in patients with macroalbuminuria and microalbuminuria and the serum HMW adiponectin-UAE association has been reported to be positive (Komaba et al. 2006). Taking into account the relatively low permeability of the glomerular filtration barrier for HMW adiponectin compared with the other subforms of adiponectin, serum HMW adiponectin levels are expected to best reflect the production of adiponectin by adipose tissue. In this aspect, the positive circulating HMW adiponectin-UAE relationship could reflect the compensatory upregulation of adiponectin production in response to albuminuria. Although HMW adiponectin has been proven to mediate predominantly the insulin-sensitising effects of adiponectin, the low permeability of the glomerular filtration barrier for HMW adiponectin indicates that HMW adiponectin may not have considerable renoprotective effects. Further studies are needed to elucidate which is the most biologically active fraction of adiponectin regarding its renoprotective effects.
The above-mentioned pathophysiological interplay between adiponectin and albuminuria can explain the reported renoprotective effects of HDL. Specifically, observational studies have shown that high HDL-C is protective against the development of albuminuria in patients with or without diabetes (O'Seaghdha et al. 2010). However, causality studies investigating the possible direct influence of HDL on renal function and development of albuminuria are lacking. There is a bidirectional causal relationship between HDL and adiponectin (HDL induces adiponectin production and vice versa) recently described by our study group and a well-known positive relationship between HDL-C and circulating adiponectin (Christou \& Kiortsis 2013). Thus, a possible mechanism explaining, at least in part, the prognostic value of high HDL-C against development of albuminuria is that HDL-C may constitute a marker of the renoprotective effects of adiponectin.

An important limitation in most of the studies investigating the adiponectin-UAE relationship was that these studies included subjects receiving drugs affecting UAE, such as ACE-I and ARBs. Taking into account the use of ACE-I and ARBs preferentially in subjects with advanced albuminuria to reduce further increases in $\mathrm{UAE}$, these drugs could have confounded the adiponectin-UAE relationship. Another limitation of some studies investigating the relationship between adiponectin and UAE is that patients with previously persistent macroalbuminuria, but with $\mathrm{UAE}<300 \mathrm{mg} / 24 \mathrm{~h}$ at the time of investigation (due to appropriate treatment), were analysed as having macroalbuminuria (Jorsal et al. 2013). In addition, the investigation of adiponectin-albuminuria relationship has been performed mostly in diabetics. Therefore the presumed role of adiponectin with regard to albuminuria in nondiabetic individuals is majorly based on the extrapolation of data derived from studies with diabetics.

\section{Adiponectin concentration and levels of adiponectin receptors in injured kidneys}

Decreased glomerular endothelial staining for adiponectin has been reported in patients with diabetic nephropathy (von Eynatten et al. 2009). Similarly, renal tissue from cases of systemic lupus erythematosus showed markedly decreased or absent endothelial staining for adiponectin in areas of glomerular hypercellularity or sclerosis and interstitial inflammation (Rovin et al. 2005). A plausible mechanism explaining these findings is that renal stress together with loss of endothelial binding sites for adiponectin form the injured glomerular capillary wall may promote shedding of adiponectin from endothelial

Published by Bioscientifica Ltd 
surfaces by proteolytic cleavage, causing degradation of high-order complexes of adiponectin and subsequent appearance of adiponectin monomers, dimers and trimers in urine (Fig. 1). In this instance, the lower the adiponectin concentration is in injured kidneys the greater the loss of beneficial renal effects of adiponectin (Rovin et al. 2005, von Eynatten et al. 2009). Because there are limited available data regarding the distribution pattern of glomerular adiponectin in different renal pathologies, further studies are needed to clarify this issue. In addition, rats with adenine-induced chronic renal failure had increased serum and urinary adiponectin levels and increased renal expression of Adipor1 and Adipor2 (Yu et al. 2013). Furthermore, renal Adipor1-mRNA and Adipor2-mRNA levels were positively correlated with serum and urinary adiponectin levels (Yu et al. 2013). These findings indicate that renal injury may cause a compensatory upregulation of ADIPOR1 and ADIPOR2 receptors in kidneys to mitigate further renal injury (Fig. 1).

\section{The role of adiponectin in obesity-induced albuminuria}

Obesity per se can induce renal injury resulting in albuminuria, independently of the presence of diabetes and hypertension which are often associated with obesity (Kiortsis \& Christou 2012). Specifically, the renal effects of obesity can be divided into structural and functional effects. The structural changes include the obesity-related glomerulopathy, which is characterised by focal segmental glomerulosclerosis, glomerulomegaly, mesangial hyperplasia and minimal foot process fusion. The functional changes include an initial progressive increase in both renal blood flow and GFR, accompanied by increased UAE and progressive reduction in GFR in later stages of the disease paralleled by worsening histological lesions and further increases in UAE. Taking into account the beneficial effects of adiponectin on renal structure and function mentioned earlier, the downregulation of adiponectin in obesity may constitute a plausible mechanism explaining, at least in part, the development of albuminuria in obese patients. Consistently, the data from the study of Ohashi et al. (2007) previously mentioned further reinforce this proposed mechanism. This study demonstrated that adiponectin could attenuate the adverse renal effects, including albuminuria, that were caused by partial nephrectomy. The state of subtotal nephrectomy in this study is relevant enough to the mismatch of nephron number to body size (with subsequent glomerular hyperfiltration) encountered in obesity (Ohashi et al. 2007).
Therefore, adiponectin possibly protects from the development of the obesity-induced adverse glomerular changes and progression to albuminuria.

The negative relationship between circulating adiponectin and UAE in the context of normoalbuminuria or lowgrade microalbuminuria was consistently found in obese populations, while this association was inconsistently reported in nonobese subjects and when it did exist this association was too weak (Yano et al. 2007, Barlovic et al. 2010, Tamba et al. 2010, Vasilescu \& Ionescu-Tîrgovişte 2011). The underlying mechanism for these associations has not been clarified yet. A plausible explanation for these data is that the protective role of adiponectin against the development of albuminuria in the early stages of this process is possibly more pronounced in obese subjects, in whom the considerable downregulation of circulating adiponectin may render adiponectin more crucial for protection against the development of albuminuria.

\section{The role of adiponectin in diabetes-induced albuminuria}

The well-known downregulation of serum adiponectin levels in patients with type 2 diabetes (Hotta et al. 2000) possibly predisposes these individuals to the development of albuminuria, because of decreased renoprotective effects of adiponectin. This mechanism may, at least in part, account for the frequent existence of albuminuria in patients with even early diabetic nephropathy. Furthermore, the baseline levels of serum adiponectin have been reported to be negatively correlated to the change in UAE over long-term follow-up of patients with type 2 diabetes (Kacso et al. 2012b). Thus, the lower the circulating adiponectin the higher the probability of future worsening of albuminuria. In addition, the upregulation of circulating adiponectin in type 2 diabetics with advanced albuminuria as mentioned above conflicts with the usual insulin resistance-induced downregulation of circulating adiponectin in patients with type 2 diabetes. This mechanism can explain the reported paradoxical upregulation or absence of difference of circulating adiponectin in type 2 diabetics with nephropathy compared with nondiabetics (Looker et al. 2004, Fujita et al. 2006). Moreover, taking into account the progressive deterioration of diabetic renal disease over time, this mechanism could also explain, at least in part, the fact that serum adiponectin levels increase with increasing diabetes duration in patients with type 2 diabetes (Looker et al. 2004). An incremental upregulation of serum adiponectin levels with increasing UAE from normoalbuminuria to

Published by Bioscientifica Ltd 
macroalbuminuria was found in diabetics with various durations of diabetes (Looker et al. 2004). This relationship persisted even after adjustment for diabetes duration.

It should be noted that there are difficulties in investigating the changes in serum adiponectin levels in response to albuminuria in diabetics due to the type 2 diabetes-associated downregulation of serum adiponectin levels (Hotta et al. 2000) and type 1 diabetes-associated upregulation of serum adiponectin levels (Frystyk et al. 2005). In addition, the investigation of the adiponectinalbuminuria association is quite challenging in diabetics with advanced albuminuria because of the frequently coexistent decreased GFR that tends to elevate circulating adiponectin. Thus, definite conclusions about the role of adiponectin with regard to albuminuria in the context of advanced diabetic renal disease are very difficult to draw, to the extent that albuminuria cannot be separately investigated from decreased GFR in this setting. In addition, in studies investigating the relationship between adiponectin and albuminuria relationship in diabetics the affected individuals were receiving antidiabetics that could influence circulating adiponectin, such as thiazolidinediones that are known to increase serum adiponectin levels (Yu et al. 2002). This factor could hamper the investigation of the albuminuria-induced regulation of serum adiponectin levels.

\section{The prediction of CVD by adiponectin in subjects with renal disease}

Taking into account the antiatherogenic and cardioprotective properties of adiponectin, high serum adiponectin levels are expected to be prognostic of low cardiovascular risk (Hotta et al. 2000). The reported property of adiponectin to protect against the development of albuminuria may, at least in part, account for the reported association of decreased serum adiponectin levels with increased cardiovascular risk, because albuminuria constitutes a well-known risk factor for CVD (de Jong \& Curhan 2006). However, data from studies investigating patients with renal disease do not follow consistently the high circulating adiponectin-low cardiovascular risk pattern. Specifically, regarding patients with renal disease, there are not only studies reporting that high serum adiponectin levels can predict decreased mortality and low future cardiovascular risk (Zoccali et al. 2002, Becker et al. 2005, Costacou et al. 2005, Abdallah et al. 2012), but there are also studies showing that high serum adiponectin levels are associated with increased mortality and incidence of CVD (Frystyk et al. 2005, Menon et al. 2006, Schalkwijk et al. 2006, Jorsal et al. 2008, Ohashi et al. 2008).
The latter pattern was reported mostly in cases with excessive upregulation of circulating adiponectin (above $15 \mathrm{mg} / \mathrm{l}$ and usually at least $20 \mathrm{mg} / \mathrm{l}$ ), while the former one was found exclusively in subjects with smaller increases in serum adiponectin levels (below $20 \mathrm{mg} / \mathrm{l}$ and usually $<15 \mathrm{mg} / \mathrm{l})$. A possible explanation for these data is that advanced renal disease not only elevates cardiovascular risk but also increases circulating adiponectin either due to reduced renal clearance of adiponectin or due to the counteracting of increased production of adiponectin to mitigate the highly atherogenic uremic milieu and/or albuminuria. This pattern possibly becomes more prevalent with increasing serum adiponectin values, overwhelming the reverse pattern of high circulating adiponectin-low cardiovascular risk. In this aspect, in patients with renal disease and serum adiponectin levels above $20 \mathrm{mg} / \mathrm{l}$, the paradoxical pattern (high serum adiponectin-high cardiovascular risk) is expected to exist, while in case of serum adiponectin levels below $15 \mathrm{mg} / \mathrm{l}$ the reverse pattern is prevalent.

Not only plasma adiponectin levels but also urinary adiponectin excretion rate appear to be novel cardiovascular risk factors. Specifically, inclusion of urinary adiponectin excretion rate added significant predictive value for predicting carotid intima-media thickness (IMT) in type 2 diabetics with normoalbuminuria or microalbuminuria compared with the basic model of the United Kingdom Prospective Diabetes Study coronary heart disease (UKPDS CHD) risk engine factors (von Eynatten et al. 2009). However, addition of UAE to the basic model did not significantly alter the predictive value for IMT. These findings imply that the urinary adiponectin excretion rate may be superior to UAE for the prediction of increased IMT. Although microalbuminuria is currently used as a marker of endothelial dysfunction and can predict cardiovascular risk in type 2 diabetics, albumin appears in urine after diabetes durations of at least 6 years and vascular changes start long before the appearance of microalbuminuria (Gerstein et al. 2000, de Jong \& Curhan 2006). Therefore, applicability of microalbuminuria for cardiovascular risk evaluation in type 2 diabetes per se appears to be limited. Importantly, significant adiponectinuria has been reported in type 2 diabetics with increased IMT but without albuminuria (von Eynatten et al. 2009). From this point of view, adiponectinuria could be an early marker of vascular damage on a prealbuminuric level of diabetic nephropathy, and the urinary adiponectin excretion rate may exceed the predictive value of UAE for cardiovascular risk evaluation. Further prospective studies are necessary to confirm these associations.

Published by Bioscientifica Ltd 


\section{The role of adiponectin in medical interventions influencing albuminuria}

Treatment with ACE-I or ARBs has been reported to increase serum adiponectin levels in the majority of the related studies (Furuhashi et al. 2003, Yenicesu et al. 2005, Schalkwijk et al. 2006). Therefore, blockade of the renninangiotensin system (RAS) appears to increase circulating adiponectin. The relevant presumptive mechanisms of increased adiponectin production involve improvement in insulin resistance and inhibition of TNF $\alpha$ secretion, but are based mostly on indirect data (Furuhashi et al. 2003, Yenicesu et al. 2005). The upregulation of circulating adiponectin by RAS blockade and the above-mentioned adiponectin-induced attenuation of adverse renal effects of angiotensin II (Fang et al. 2013) could explain, at least in part, the well-known RAS blockade-induced improvement in insulin sensitivity and protection against the development of albuminuria (Furuhashi et al. 2003, 2004).

Thiazolidinediones have been shown to decrease UAE in diabetics and the reduction in UAE has been associated with the thiazolidinediones-induced increase in serum adiponectin levels (Miyazaki et al. 2007). These findings indicate that the beneficial effects of thiazolidinediones on diabetic nephropathy may be mediated by the thiazolidinediones-induced upregulation of circulating adiponectin which is PPAR $\gamma$-dependent. Consistently, elevated serum adiponectin levels in $\operatorname{PPAR} \gamma$-agonist treated mice appear to increase recovery of injured podocytes (Rutkowski et al. 2013).

Fenofibrate treatment decreases UAE, but the exact underlying mechanism remains unknown (Keech et al. 2005). Moreover, fenofibrate has been reported to increase adiponectin expression in adipose tissue and serum adiponectin levels through PPAR $\alpha$ activation and HDL elevation (Christou \& Kiortsis 2013). Thus, the fenofibrate-induced elevation of serum adiponectin levels may contribute to the concomitant decrease in UAE, because of the renoprotective effects of adiponectin. Consistently, unpublished data from a previous trial performed by our study group showed that the percentage change in circulating HMW adiponectin over 3 months of fenofibrate treatment was negatively correlated with the percentage change in urinary albumin to creatinine ratio (ACR) $(r=-0.648, P=0.043)$ (Christou et al. 2012).

Weight loss can induce a decrease in UAE in overweight or obese subjects (Kiortsis \& Christou 2012). Longterm diets have been shown to increase serum adiponectin levels (Christou \& Kiortsis 2013), indicating a possible role of adiponectin on mediating, at least in part, the weight loss-induced changes of UAE. There is a study mentioning that the change in serum adiponectin levels over 1 year of diet was not significantly correlated with the change in ACR (Nakatsuji et al. 2010). Unpublished data from a previous trial performed by our study group showed that circulating HMW adiponectin at baseline was negatively correlated with the percentage change of logACR over 3 months of diet $(r=-0.769, P=0.003)$ (Christou et al. 2012). Thus, high baseline serum adiponectin levels may predict a more favourable response of UAE during diet, possibly reflecting a higher grade of nephroprotection. Further studies are needed to elucidate the possible role of adiponectin in the weight-loss-induced decrease in UAE.

\section{Conclusion}

Adiponectin is an adipokine that appears to have renoprotective effects through AMPK-activated pathways, resulting in the prevention of albuminuria. The relationship between circulating adiponectin and albuminuria has been reported to show a biphasic pattern. A negative relationship exists in the case of normoalbuminuria, possibly reflecting the increased renal clearance of adiponectin together with the renoprotective effects of adiponectin, and a positive association in more advanced albuminuria, indicating the counteracting upregulation of serum adiponectin levels to mitigate renal injury. Taking into account the fact that the currently believed route of renal biodegradation/excretion of adiponectin is not based on firm evidence, the confirmation of this route and the exploration of the exact related pathways are prerequisites for understanding the role of adiponectin with regard to renal physiology. In addition, the accepted renoprotective role of adiponectin has been based almost exclusively on data from nonhuman experiments, necessitating the performance of studies investigating the relevant issues in human experimental settings. Further clinical studies are needed to translate convincing experimental evidence for the nephroprotective role of adiponectin into clinical practice that could create new therapies for albuminuria, targeting specific pathways of adiponectin action.

Declaration of interest

The authors declare that there is no conflict of interest that could be perceived as prejudicing the impartiality of the review.

\section{Funding}

This research did not receive any specific grant from any funding agency in the public, commercial or not-for-profit sector.

Published by Bioscientifica Ltd. 


\section{References}

Abdallah E, Waked E, Nabil M \& El-Bendary O 2012 Adiponectin and cardiovascular outcomes among hemodialysis patients. Kidney \& Blood Pressure Research 35 247-253. (doi:10.1159/000334649)

Barlovic DP, Zaletel J \& Prezelj J 2009 Adipocytokines are associated with renal function in patients with normal range glomerular filtration rate and type 2 diabetes. Cytokine 46 142-145. (doi:10.1016/j.cyto. 2009.01.001)

Barlovic DP, Zaletel J \& Prezelj J 2010 Association between adiponectin and low-grade albuminuria is BMI-dependent in type 2 diabetes. Kidney \& Blood Pressure Research 33 405-410. (doi:10.1159/000318292)

Becker B, Kronenberg F, Kielstein JT, Haller H, Morath C, Ritz E, Fliser D \& MMKD Study Group 2005 Renal insulin resistance syndrome, adiponectin and cardiovascular events in patients with kidney disease: the mild and moderate kidney disease study. Journal of the American Society of Nephrology 16 1091-1098. (doi:10.1681/ASN.2004090742)

Cammisotto PG \& Bendayan M 2008 Adiponectin stimulates phosphorylation of AMP-activated protein kinase $\alpha$ in renal glomeruli. Journal of Molecular Histology 39 579-584. (doi:10.1007/s10735-008-9198-6)

Cheng CF, Lian WS, Chen SH, Lai PF, Li HF, Lan YF, Cheng WT \& Lin H 2012 Protective effects of adiponectin against renal ischemiareperfusion injury via prostacyclin-PPAR $\alpha$-heme oxygenase-1 signaling pathway. Journal of Cellular Physiology 227 239-249. (doi:10.1002/ jcp.22726)

Christou GA \& Kiortsis DN 2013 Adiponectin and lipoprotein metabolism. Obesity Reviews 14 939-949. (doi:10.1111/obr.12064)

Christou GA, Tellis KC, Elisaf MC, Tselepis AD \& Kiortsis DN 2012 High density lipoprotein is positively correlated with the changes in circulating total adiponectin and high molecular weight adiponectin during dietary and fenofibrate treatment. Hormones 11 178-188. (doi:10.14310/horm.2002.1345)

Costacou T, Zgibor JC, Evans RW, Otvos J, Lopes-Virella MF, Tracy RP \& Orchard TJ 2005 The prospective association between adiponectin and coronary artery disease among individuals with type 1 diabetes. The Pittsburgh Epidemiology of Diabetes Complications Study. Diabetologia 48 41-48. (doi:10.1007/s00125-004-1597-y)

Davis BJ, Xie Z, Viollet B \& Zou MH 2006 Activation of the AMP-activated kinase by antidiabetes drug metformin stimulates nitric oxide synthesis in vivo by promoting the association of heat shock protein 90 and endothelial nitric oxide synthase. Diabetes 55 496-505. (doi:10.2337/ diabetes.55.02.06.db05-1064)

von Eynatten M, Liu D, Hock C, Oikonomou D, Baumann M, Allolio B, Korosoglou G, Morcos M, Campean V, Amann K et al. 2009 Urinary adiponectin excretion: a novel marker for vascular damage in type 2 diabetes. Diabetes 58 2093-2099. (doi:10.2337/db09-0204)

Fang F, Liu GC, Kim C, Yassa R, Zhou J \& Scholey JW 2013 Adiponectin attenuates angiotensin II-induced oxidative stress in renal tubular cells through AMPK and cAMP-Epac signal transduction pathways. American Journal of Physiology. Renal Physiology 304 F1366-F1374. (doi:10.1152/ ajprenal.00137.2012)

Frystyk J, Tarnow L, Hansen TK, Parving HH \& Flyvbjerg A 2005 Increased serum adiponectin levels in type 1 diabetic patients with microvascular complications. Diabetologia 48 1911-1918. (doi:10.1007/s00125-0051850-z)

Fujita H, Morii T, Koshimura J, Ishikawa M, Kato M, Miura T, Sasaki H, Narita T, Ito S \& Kakei M 2006 Possible relationship between adiponectin and renal tubular injury in diabetic nephropathy. Endocrine Journal 53 745-752. (doi:10.1507/endocrj.K06-016)

Furuhashi M, Ura N, Higashiura K, Murakami H, Tanaka M, Moniwa N, Yoshida D \& Shimamoto K 2003 Blockade of the renin-angiotensin system increases adiponectin concentrations in patients with essential hypertension. Hypertension 42 76-81. (doi:10.1161/01.HYP. $0000078490.59735 .6 \mathrm{E})$
Furuhashi M, Ura N, Takizawa H, Yoshida D, Moniwa N, Murakami H, Higashiura K \& Shimamoto K 2004 Blockade of the renin-angiotensin system decreases adipocyte size with improvement in insulin sensitivity. Journal of Hypertension 22 1977-1982. (doi:10.1097/00004872200410000-00021)

Gerstein HC, Mann JF, Pogue J, Dinneen SF, Hallé JP, Hoogwerf B, Joyce C, Rashkow A, Young J, Zinman B et al. 2000 Prevalence and determinants of microalbuminuria in high-risk diabetic and nondiabetic patients in the Heart Outcomes Prevention Evaluation Study. Diabetes Care 23(Suppl 2) B35-B39.

Halberg N, Schraw TD, Wang ZV, Kim JY, Yi J, Hamilton MP, Luby-Phelps K \& Scherer PE 2009 Systemic fate of the adipocyte-derived factor adiponectin. Diabetes 58 1961-1970. (doi:10.2337/db08-1750)

Hayakawa K, Ohashi H, Yokoyama H, Yoshida G, Okada M \& Minatoguchi S 2009 Adiponectin is increased and correlated with the degree of proteinuria, but plasma leptin is not changed in patients with chronic glomerulonephritis. Nephrology 14 327-331. (doi:10.1111/j.1440-1797. 2008.01047.x)

Hotta K, Funahashi T, Arita Y, Takahashi M, Matsuda M, Okamoto Y, Iwahashi $\mathrm{H}$, Kuriyama H, Ouchi N, Maeda K et al. 2000 Plasma concentrations of a novel, adipose-specific protein, adiponectin, in type 2 diabetic patients. Arteriosclerosis, Thrombosis, and Vascular Biology 20 1595-1599. (doi:10.1161/01.ATV.20.6.1595)

Isobe T, Saitoh S, Takagi S, Takeuchi H, Chiba Y, Katoh N \& Shimamoto K 2005 Influence of gender, age and renal function on plasma adiponectin level: the Tanno and Sobetsu study. European Journal of Endocrinology 153 91-98. (doi:10.1530/eje.1.01930)

Jian DY, Chao YW, Ting CH, Huang SW, Chang CF, Juan CC \& Chen JY 2013 Losartan ameliorates renal injury, hypertension, and adipocytokine imbalance in 5/6 nephrectomized rats. European Journal of Pharmacology 709 85-92. (doi:10.1016/j.ejphar.2013.03.024)

Jin X, Chen J, Hu Z, Chan L \& Wang Y 2013 Genetic deficiency of adiponectin protects against acute kidney injury. Kidney International 83 604-614. (doi:10.1038/ki.2012.408)

de Jong PE \& Curhan GC 2006 Screening, monitoring, and treatment of albuminuria: public health perspectives. Journal of the American Society of Nephrology 17 2120-2126. (doi:10.1681/ASN.2006010097)

Jorsal A, Tarnow L, Frystyk J, Lajer M, Flyvbjerg A, Parving HH, Vionnet N \& Rossing P 2008 Serum adiponectin predicts all-cause mortality and end stage renal disease in patients with type I diabetes and diabetic nephropathy. Kidney International 74 649-654. (doi:10.1038/ki. 2008.201)

Jorsal A, Petersen EH, Tarnow L, Hess G, Zdunek D, Frystyk J, Flyvbjerg A, Lajer M \& Rossing P 2013 Urinary adiponectin excretion rises with increasing albuminuria in type 1 diabetes. Journal of Diabetes and its Complications 27 604-608. (doi:10.1016/j.jdiacomp.2013.06.008)

Kacso IM, Bondor CI \& Kacso G 2012a Plasma adiponectin is related to the progression of kidney disease in type 2 diabetes patients. Scandinavian Journal of Clinical and Laboratory Investigation 72 333-339. (doi:10.3109/ 00365513.2012.668928)

Kacso IM, Trifa AP, Popp RA \& Kacso G 2012b Association of 276G > T adiponectin gene polymorphism to plasma adiponectin and albuminuria in type 2 diabetic patients. International Urology and Nephrology $\mathbf{4 4}$ 1771-1777. (doi:10.1007/s11255-011-0118-4)

Kamimura MA, Canziani ME, Sanches FR, Velludo CM, Carrero JJ, Bazanelli AP, Draibe SA \& Cuppari L 2012 Variations in adiponectin levels in patients with chronic kidney disease: a prospective study of 12 months. Jornal Brasileiro de Nefrologia 34 259-265. (doi:10.5935/0101-2800. 20120007)

Keech A, Simes RJ, Barter P, Best J, Scott R, Taskinen MR, Forder P, Pillai A, Davis T, Glasziou P et al. 2005 Effects of long-term fenofibrate therapy on cardiovascular events in 9795 people with type 2 diabetes mellitus (the FIELD study): randomised controlled trial. Lancet 366 1849-1861. (doi:10.1016/S0140-6736(05)67667-2)

Published by Bioscientifica Ltd 
Kiortsis DN \& Christou MA 2012 Management of obesity-induced kidney disease: a critical review of the literature. Obesity Facts 5 821-832. (doi:10.1159/000345919)

Komaba H, Igaki N, Goto S, Yokota K, Doi H, Takemoto T, Kohno M, Hirosue Y, Goto T et al. 2006 Increased serum high-molecular-weight complex of adiponectin in type 2 diabetic patients with impaired renal function. American Journal of Nephrology 26 476-482. (doi:10.1159/ 000096870)

Komura N, Kihara S, Sonoda M, Maeda N, Tochino Y, Funahashi T \& Shimomura I 2010 Increment and impairment of adiponectin in renal failure. Cardiovascular Research 86 471-477. (doi:10.1093/cvr/cvp415)

Koshimura J, Fujita H, Narita T, Shimotomai T, Hosoba M, Yoshioka N, Kakei M, Fujishima H \& Ito S 2004 Urinary adiponectin excretion is increased in patients with overt diabetic nephropathy. Biochemical and Biophysical Research Communications 316 165-169. (doi:10.1016/j.bbrc. 2004.02.032)

Lee MJ, Feliers D, Mariappan MM, Sataranatarajan K, Mahimainathan L, Musi N, Foretz M, Viollet B, Weinberg JM, Choudhury GG et al. 2007 A role for AMP-activated protein kinase in diabetes-induced renal hypertrophy. American Journal of Physiology. Renal Physiology 292 F617-F627. (doi:10.1152/ajprenal.00278.2006)

Looker HC, Krakoff J, Funahashi T, Matsuzawa Y, Tanaka S, Nelson RG, Knowler WC, Lindsay RS \& Hanson RL 2004 Adiponectin concentrations are influenced by renal function and diabetes duration in Pima Indians with type 2 diabetes. Journal of Clinical Endocrinology and Metabolism 89 4010-4017. (doi:10.1210/jc.2003-031916)

Martinez Cantarin MP, Waldman SA, Doria C, Frank AM, Maley WR, Ramirez CB, Keith SW \& Falkner B 2013 The adipose tissue production of adiponectin is increased in end-stage renal disease. Kidney International 83 487-494. (doi:10.1038/ki.2012.421)

Menon V, Li L, Wang X, Greene T, Balakrishnan V, Madero M, Pereira AA, Beck GJ, Kusek JW, Collins AJ et al. 2006 Adiponectin and mortality in patients with chronic kidney disease. Journal of the American Society of Nephrology 17 2599-2606. (doi:10.1681/ASN.2006040331)

Miyazaki Y, Cersosimo E, Triplitt C \& DeFronzo RA 2007 Rosiglitazone decreases albuminuria in type 2 diabetic patients. Kidney International 72 1367-1373. (doi:10.1038/sj.ki.5002516)

Nakamaki S, Satoh H, Kudoh A, Hayashi Y, Hirai H \& Watanabe T 2011 Adiponectin reduces proteinuria in streptozotocin-induced diabetic Wistar rats. Experimental Biology and Medicine 236 614-620. (doi:10.1258/ebm.2011.010218)

Nakatsuji H, Kishida K, Funahashi T, Noguchi M, Ogawa T, Okauchi Y, Nishizawa H, Nakamura T, Matsuzawa Y \& Shimomura I 2010 One-year reductions in body weight and blood pressure, but not in visceral fat accumulation and adiponectin, improve urinary albumin-to-creatinine ratio in middle-aged Japanese men. Diabetes Care 33 e110-e111. (doi:10.2337/dc10-0739)

Obata Y, Yamada Y, Takahi Y, Baden MY, Saisho K, Tamba S, Yamamoto K, Umeda M, Furubayashi A \& Matsuzawa Y 2013 Relationship between serum adiponectin levels and age in healthy subjects and patients with type 2 diabetes. Clinical Endocrinology 79 204-210. (doi:10.1111/cen. 12041)

Ohashi K, Iwatani H, Kihara S, Nakagawa Y, Komura N, Fujita K, Maeda N, Nishida M, Katsube F, Shimomura I et al. 2007 Exacerbation of albuminuria and renal fibrosis in subtotal renal ablation model of adiponectin-knockout mice. Arteriosclerosis, Thrombosis, and Vascular Biology 27 1910-1917. (doi:10.1161/ATVBAHA.107.147645)

Ohashi N, Kato A, Misaki T, Sakakima M, Fujigaki Y, Yamamoto T \& Hishida A 2008 Association of serum adiponectin levels with all-cause mortality in hemodialysis patients. Internal Medicine 47 485-491. (doi:10.2169/internalmedicine.47.0614)

O'Seaghdha CM, Hwang SJ, Upadhyay A, Meigs JB \& Fox CS 2010 Predictors of incident albuminuria in the Framingham Offspring cohort. American Journal of Kidney Diseases 56 852-860. (doi:10.1053/j.ajkd.2010.04.013)
Ouedraogo R, Wu X, Xu SQ, Fuchsel L, Motoshima H, Mahadev K, Hough K, Scalia R \& Goldstein BJ 2006 Adiponectin suppression of high-glucoseinduced reactive oxygen species in vascular endothelial cells: evidence for involvement of a cAMP signaling pathway. Diabetes 55 1840-1846. (doi:10.2337/db05-1174)

Perri A, Vizza D, Lofaro D, Gigliotti P, Leone F, Brunelli E, Malivindi R, De Amicis F, Romeo F, De Stefano R et al. 2013 Adiponectin is expressed and secreted by renal tubular epithelial cells. Journal of Nephrology 26 1049-1054. (doi:10.5301/jn.5000269)

Qi L, Li T, Rimm E, Zhang C, Rifai N, Hunter D, Doria A \& Hu FB 2005 The +276 polymorphism of the APM1 gene, plasma adiponectin concentration, and cardiovascular risk in diabetic men. Diabetes 54 1607-1610. (doi:10.2337/diabetes.54.5.1607)

Rakatzi I, Mueller H, Ritzeler O, Tennagels N \& Eckel J 2004 Adiponectin counteracts cytokine- and fatty acid-induced apoptosis in the pancreatic $\beta$-cell line INS-1. Diabetologia 47 249-258. (doi:10.1007/ s00125-003-1293-3)

Rovin BH, Song H, Hebert LA, Nadasdy T, Nadasdy G, Birmingham DJ, Yung Yu C \& Nagaraja HN 2005 Plasma, urine, and renal expression of adiponectin in human systemic lupus erythematosus. Kidney International 68 1825-1833. (doi:10.1111/j.1523-1755.2005.00601.x)

Rutkowski JM, Wang ZV, Park AS, Zhang J, Zhang D, Hu MC, Moe OW, Susztak K \& Scherer PE 2013 Adiponectin promotes functional recovery after podocyte ablation. Journal of the American Society of Nephrology $\mathbf{2 4}$ 268-282. (doi:10.1681/ASN.2012040414)

Schalkwijk CG, Chaturvedi N, Schram MT, Fuller JH, Stehouwer CD \& EURODIAB Prospective Complications Study Group 2006 Adiponectin is inversely associated with renal function in type 1 diabetic patients. Journal of Clinical Endocrinology and Metabolism 91 129-135. (doi:10.1210/jc.2005-1117)

Sharma K, Ramachandrarao S, Qiu G, Usui HK, Zhu Y, Dunn SR, Ouedraogo R, Hough K, McCue P, Chan L et al. 2008 Adiponectin regulates albuminuria and podocyte function in mice. Journal of Clinical Investigation 118 1645-1656. (doi:10.1172/JCI32691)

Shen YY, Hughes JT, Charlesworth JA, Kelly JJ \& Peake PW 2008 Adiponectin is present in the urine in its native conformation, and specifically reduces the secretion of MCP-1 by proximal tubular cells. Nephrology 13 405-410. (doi:10.1111/j.1440-1797.2008.00949.x)

Shimotomai T, Kakei M, Narita T, Koshimura J, Hosoba M, Kato M, Komatsuda A \& Ito S 2005 Enhanced urinary adiponectin excretion in IgA-nephropathy patients with proteinuria. Renal Failure 27 323-328. (doi:10.1081/JDI-56597)

Tacke F, Wüstefeld T, Horn R, Luedde T, Srinivas Rao A, Manns MP, Trautwein C \& Brabant G 2005 High adiponectin in chronic liver disease and cholestasis suggests biliary route of adiponectin excretion in vivo. Journal of Hepatology 42 666-673. (doi:10.1016/j.jhep. 2004.12.024)

Takahashi M, Otsubo S, Uchida K, Yumura W \& Nitta K 2007 Association between serum adiponectin levels and arteriolosclerosis in IgA nephropathy patients. Internal Medicine 46 453-459. (doi:10.2169/ internalmedicine.46.6332)

Tamba S, Nakatsuji H, Kishida K, Noguchi M, Ogawa T, Okauchi Y, Nishizawa H, Imagawa A, Nakamura T, Matsuzawa Y et al. 2010 Relationship between visceral fat accumulation and urinary albumincreatinine ratio in middle-aged Japanese men. Atherosclerosis 211 601-605. (doi:10.1016/j.atherosclerosis.2010.02.037)

Tsioufis C, Dimitriadis K, Chatzis D, Vasiliadou C, Tousoulis D, Papademetriou V, Toutouzas P, Stefanadis C \& Kallikazaros I 2005 Relation of microalbuminuria to adiponectin and augmented C-reactive protein levels in men with essential hypertension. American Journal of Cardiology 96 946-951. (doi:10.1016/j.amjcard.2005.05.052)

Vasilescu R \& Ionescu-Tîrgovişte C 2011 Association of albuminuria with adipokines, inflammation and insulin resistance in non-diabetic subjects. Revista Medico-Chirurgicală a Societăţii de Medici şi Naturalişti din Iaşi 115 1174-1182. 
Yano Y, Hoshide S, Ishikawa J, Hashimoto T, Eguchi K, Shimada K \& Kario K 2007 Differential impacts of adiponectin on low-grade albuminuria between obese and nonobese persons without diabetes. Journal of Clinical Hypertension 9 775-782. (doi:10.1111/j.1524-6175.2007.07321.x)

Yenicesu M, Yilmaz MI, Caglar K, Sonmez A, Eyileten T, Acikel C, Kilic S, Bingol N, Bingol S \& Vural A 2005 Blockade of the renin-angiotensin system increases plasma adiponectin levels in type-2 diabetic patients with proteinuria. Nephron. Clinical Practice 99 c115-c121. (doi:10.1159/ 000083929)

Yilmaz MI, Sonmez A, Caglar K, Gok DE, Eyileten T, Yenicesu M, Acikel C, Bingol N, Kilic S, Oguz Y et al. 2004 Peroxisome proliferator-activated receptor $\gamma($ PPAR- $\gamma$ ) agonist increases plasma adiponectin levels in type 2 diabetic patients with proteinuria. Endocrine 25 207-214. (doi:10.1385/ENDO:25:3:207)

Yu JG, Javorschi S, Hevener AL, Kruszynska YT, Norman RA, Sinha M \& Olefsky JM 2002 The effect of thiazolidinediones on plasma adiponectin levels in normal, obese, and type 2 diabetic subjects Diabetes 51 2968-2974. (doi:10.2337/diabetes.51.10.2968)

Yu Y, Bao BJ, Fan YP, Shi L \& Li SQ 2013 Changes of adiponectin and its receptors in rats following chronic renal failure. Renal Failure 36 92-97. (doi:10.3109/0886022X.2013.830975)

Zoccali C, Mallamaci F, Tripepi G, Benedetto FA, Cutrupi S, Parlongo S, Malatino LS, Bonanno G, Seminara G, Rapisarda F et al. 2002 Adiponectin, metabolic risk factors, and cardiovascular events among patients with end-stage renal disease. Journal of the American Society of Nephrology 13 134-141. (doi:10.1097/01.ASN.0000032548. 18973.0F)

Zoccali C, Mallamaci F, Panuccio V, Tripepi G, Cutrupi S, Parlongo S, Catalano F, Tanaka S, Ouchi N, Kihara S et al. 2003 Adiponectin is markedly increased in patients with nephrotic syndrome and is related to metabolic risk factors. Kidney International. Supplement 84 S98-102. (doi:10.1046/j.1523-1755.63.s84.49.x)

Received in final form 8 January 2014

Accepted 23 January 2014

Accepted Preprint published online 24 January 2014
Published by Bioscientifica Ltd. 